\title{
A 6-bit 800-MS/s Pipelined A/D Converter With Open-Loop Amplifiers
}

\author{
Ding-Lan Shen, Student Member, IEEE, and Tai-Cheng Lee, Member, IEEE
}

\begin{abstract}
A 6-bit 800-MS/s pipelined A/D converter (ADC) achieves SNDR and SFDR of $33.7 \mathrm{~dB}$ and $47.5 \mathrm{~dB}$, respectively. Employing voltage-mode open-loop amplifiers in gain stages, global gain control techniques, and two-bank-interleaved architecture, the proposed pipelined A/D converter relaxes stringent design tradeoffs between speed and power. Fabricated in a 0.18- $\mu \mathrm{m}$ CMOS technology, the ADC consumes $105 \mathrm{~mW}$ from a $1.8-\mathrm{V}$ power supply while the active area is only $0.5 \mathrm{~mm}^{2}$.
\end{abstract}

Index Terms-Analog-digital conversion, CMOS analog integrated circuits, gain control.

\section{INTRODUCTION}

$\mathbf{S}$ TATE-OF-THE-ART read channels in high-performance serial-link systems such as DVD disk drives and communication networks require high-speed ADCs to digitize the partial response signal for digital processing [1]. High-speed, low-resolution ADCs are dominated by flash architecture [2], [3]. Employing digital-circuit-like comparators, flash ADCs achieve high-speed conversion rates for a given technology but at the cost of higher power consumption. Pipelined ADCs can meet the needs of applications requiring medium-speed conversion rates, while improving power efficiency. One salient advantage of a pipelined ADC over a flash architecture is its low input capacitance, thereby relaxing the design requirement of preceding stage. The conversion speed of the conventional pipelined ADC architecture is limited by the need for high-gain and wide-bandwidth closed-loop amplifiers in the gain stages. Moreover, decreasing supply voltages in deep-submicron technologies restrain the design methodology of conventional high-gain amplifiers, such as cascode opamps. To overcome these barriers, several ADC designs applying open-loop amplifiers with digital calibration have been developed. In [4], an efficient method reduces the power consumption by replacing a closed-loop amplifier with an open-loop amplifier in the first stage of the pipelined ADC. References [5] and [6] use an ADC array to achieve GSample/s with current-mode open-loop amplifiers in pipelined stages.

To obtain high-speed conversion, time-interleaved techniques are prevalent in ADC designs. A time-interleaved ADC based

Manuscript received January 7, 2006; revised August 3, 2006. This work was supported by Mediatek and the National Science Council, Taiwan, under Contract 95-2220-E-002-023-.

D.-L. Shen is with the Graduate Institute of Electronics Engineering, National Taiwan University, Taipei, Taiwan 106, R.O.C.

T.-C. Lee is with the Graduate Institute of Electronics Engineering and Department of Electrical Engineering, National Taiwan University, Taipei, Taiwan, 106, R.O.C. (e-mail: tlee@cc.ee.ntu.edu.tw).

Digital Object Identifier 10.1109/JSSC.2006.889380

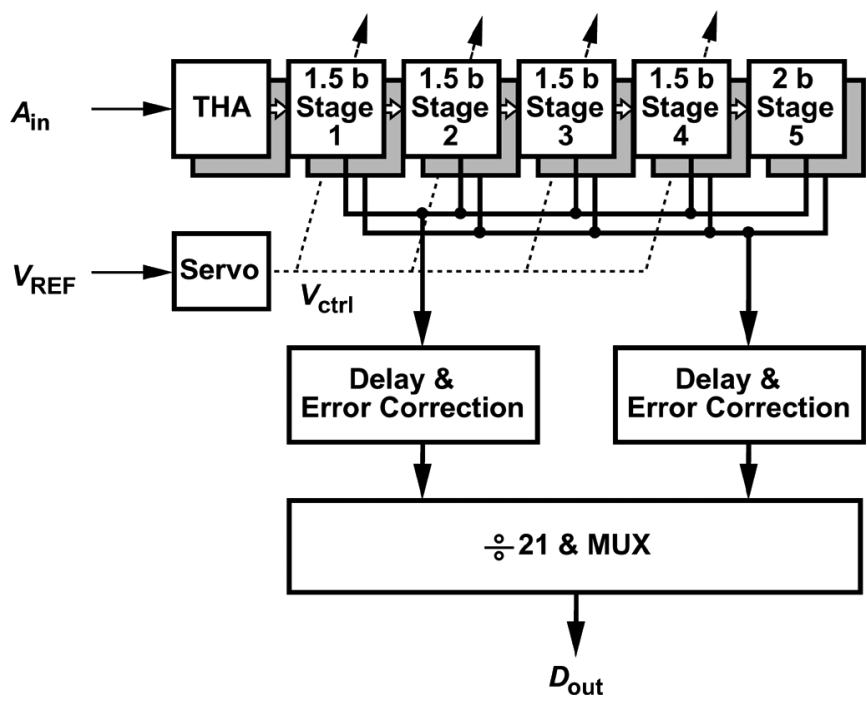

Fig. 1. System architecture.

on SAR architecture that achieves high speed and low power consumption has been presented in [7]. Pipelined ADCs with time-interleaved techniques [5], [6] can deliver even higher conversion performance than SAR ADCs [7].

In this work, a 6-bit pipelined ADC with open-loop amplifiers and a two-bank-interleaved technique are coupled to achieve the $800-\mathrm{Msample} / \mathrm{s}$ conversion rate in a $0.18-\mu \mathrm{m}$ CMOS technology. Voltage-mode open-loop amplifiers enable the converter with pipelined architecture to operate at a higher conversion rate. Without the need of digital calibration, the global gain control technique compensates the gain attenuation in open-loop amplification due to parasitic capacitances. By using the interleaved technique, this ADC achieves high-speed operation with a reasonable power level. Note that it is not beneficial to apply the stage scaling technique in the proposed ADC to improve the efficiency at the 6-bit level.

Section II introduces the system architecture of the pipelined ADC with open-loop amplifiers. In Section III, the building blocks of the ADC are described. Section IV specifies the design considerations of this ADC. Experimental results are presented in Section V, and Section VI summarizes with a conclusion.

\section{SySTEM ARCHITECTURE}

The pipelined ADC requires two nonoverlapped clocks to operate in different phases. The amplifier is in amplification mode for half of the cycle and in reset state for the other half. Shown in Fig. 1, two interleaved banks of pipelined ADC in different phases convert the sampled signal to digital data. Then, each bank of the ADC operates at $400 \mathrm{MHz}$ and the entire ADC 


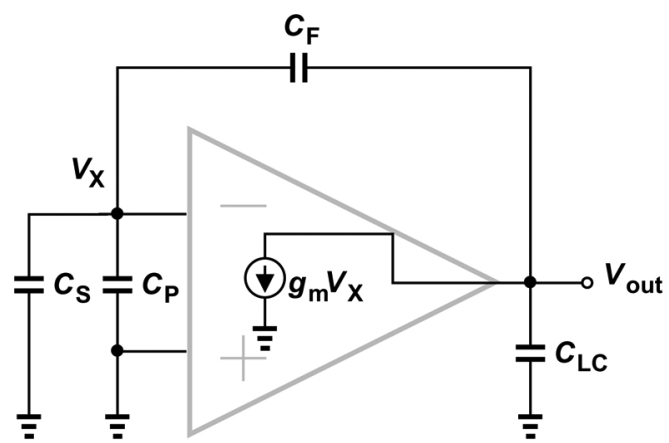

(a)

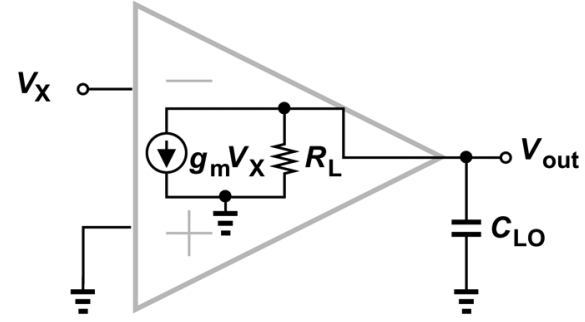

(b)

Fig. 2. (a) Closed-loop amplifier model. (b) Open-loop amplifier model.

achieves 800 Msample/s throughput, thereby relaxing the stringent bandwidth requirements of analog circuits. The track-andhold amplifier (THA) is designed for better than 8-bit linearity. The pipelined ADC chooses four 1.5-bit stages to tolerate larger comparator offset voltage. A 2-bit flash ADC is cascaded at the end of pipelined stages to construct a 6-bit pipelined ADC. With delay alignment and error correction, the ADC produces digital code at the rate of $800 \mathrm{Msample/s}$. The servo loop of the global gain control ensures gain accuracy of pipelined stages. The interleaved ADC output is decimated by 21 for data processing.

In a conventional 6-bit pipelined A/D converter design, the open-loop gain of the amplifiers in the 1.5-bit stages must be greater than $40 \mathrm{~dB}$. High-gain amplifiers require stringent design tradeoffs between power and settling time. In this work, open-loop amplifiers replace the conventional closed-loop amplifiers to achieve high-speed conversion with lower power.

Design tradeoffs between speed and power for closed-loop amplifiers and open-loop amplifiers are distinct. For a large input, the settling time of the closed-loop amplifier, an operational transconductance amplifier (OTA), may suffer from slewing at the initial time. To simplify the operation of closed-loop amplification, the model of the single-stage OTA is shown in Fig. 2(a). The transconductance of the OTA is $g_{m}$ and the maximum output current is limited to $\pm I_{\mathrm{SS}}$. The output transient response is obtained as (1) [8] for the output of the OTA changing from 0 to $V_{\text {ref }}$ :

$$
V_{\text {out }}(t)=V_{\text {ref }}+\left[V_{\text {out }}\left(t_{\text {slew }}\right)-V_{\text {ref }}\right] \cdot e^{-\left(t-t_{\text {slew }}\right) / \tau}
$$

where

$$
\begin{aligned}
V_{\text {out }}\left(t_{\text {slew }}\right)= & \frac{I_{\mathrm{SS}}}{C_{L C}+C_{F} \|\left(C_{S}+C_{P}\right)} \cdot t_{\text {slew }} \\
t_{\text {slew }}= & \frac{\left(V_{\text {ini }}-\frac{I_{\mathrm{SS}}}{g_{m}}\right) \cdot\left(C_{L C}+C_{F} \|\left(C_{S}+C_{P}\right)\right)}{I_{\mathrm{SS}}} \\
& \cdot \frac{C_{S}+C_{P}+C_{F}}{C_{F}} \\
\tau= & \frac{\left(C_{L C}+C_{F} \|\left(C_{S}+C_{P}\right)\right)}{g_{m}} \cdot \frac{C_{S}+C_{P}+C_{F}}{C_{F}}
\end{aligned}
$$

where $V_{\text {ini }}$ is the initial condition of $V_{X}$, and $t_{\text {slew }}$ is the slewing time of the OTA. When maximum output voltage is $V_{\text {ref }}$, the response of total available time $\left(t_{\text {cavl }}\right)$ for the following $N$-bit quantizer must satisfy

$$
V_{\text {out }}\left(t_{\text {cavl }}\right)=V_{\text {ref }}-\frac{2 V_{\text {ref }}}{2^{N+1}} .
$$

Equation (1) and (2) lead to

$$
t_{\text {cavl }}=t_{\text {slew }}+\tau \cdot \ln \frac{V_{\text {ref }}-V_{\text {out }}\left(t_{\text {slew }}\right)}{\left(V_{\text {ref }} / 2^{N}\right)} .
$$

The open-loop amplifier is modeled in Fig. 2(b). Because the open-loop amplifier always operates at the linear amplification region, slewing does not occur. When the output of the open-loop amplifier changes from 0 to $V_{\text {ref }}$, the output voltage is obtained as

$$
V_{\text {out }}(t)=V_{\text {ref }}\left(1-e^{-t / \tau}\right)
$$

where

$$
\tau=R_{L} \cdot C_{L O}
$$

Similarly, the total available time of the open-loop amplifier is

$$
t_{\mathrm{oavl}}=\tau \cdot \ln 2^{N}
$$

For the typical design of amplifiers, $I_{\mathrm{SS}}=1 \mathrm{~mA}, C_{S}=C_{F}=$ $300 \mathrm{fF}, C_{L C}=600 \mathrm{fF}, C_{P}=100 \mathrm{fF}, V_{\text {ini }}=100 \mathrm{mV}$, $g_{m}=11 \mathrm{~mA} / \mathrm{V}, V_{\text {ref }}=200 \mathrm{mV}$, and $N=5$, the available time of the closed-loop amplifier, $t_{\text {cavl }}$, is equal to $0.57 \mathrm{~ns}$. At the same power level, when $R_{L}=500 \Omega$ and $C_{L O}=300 \mathrm{fF}$, the available time of the open-loop amplifier, $t_{\text {oavl }}$, is equal to $0.52 \mathrm{~ns}$. Increasing $I_{\mathrm{SS}}$ or enlarging $g_{m}$ in the closed-loop amplifier reduces $t_{\text {slew }}$ but at the cost of higher power or larger parasitic capacitances and area. Moreover, stability problems arising from higher order poles must be carefully designed to avoid long settling time. Such tradeoffs present challenges in the design of high-performance amplifiers. Equation (4) indicates that the settling time of the open-loop amplifier is only the function of $R_{L}$ and $C_{L O}$. Altering these parameters to enhance the operation speed is more efficient than those in closed-loop amplifiers. Additionally, the immunity from stability consideration relaxes tradeoffs between speed and power in the open-loop amplifiers. Consequently, the simple open-loop amplifier proves 


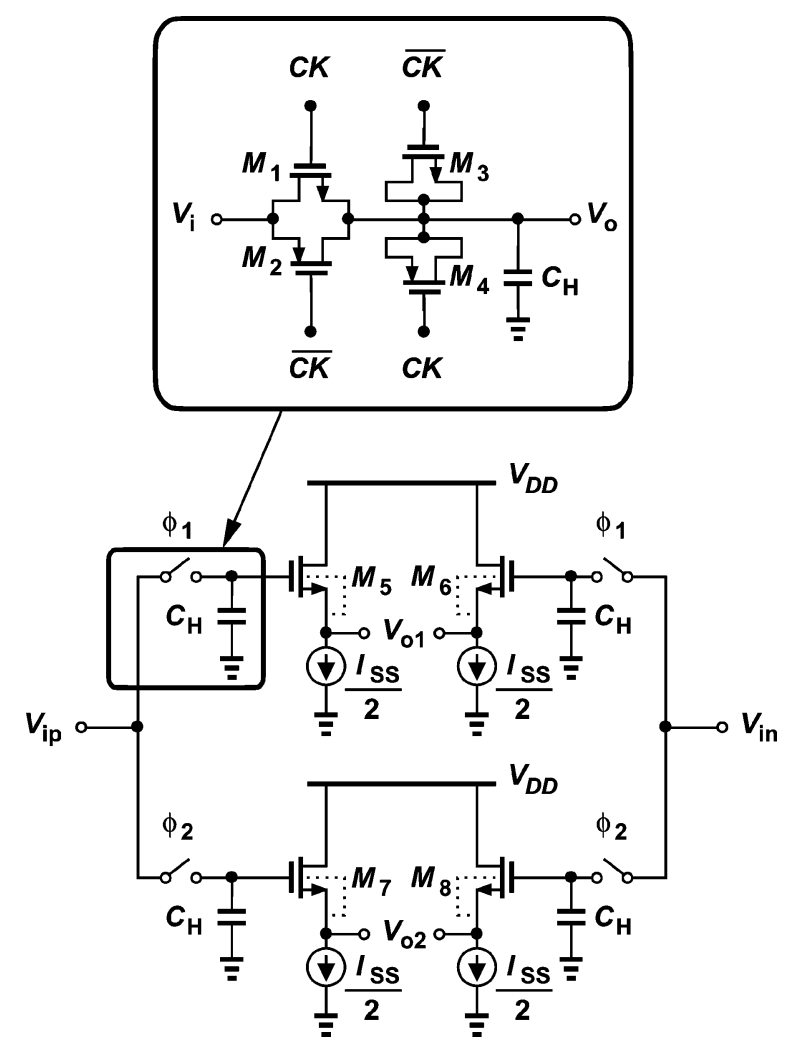

Fig. 3. Track-and-hold circuit.

a better power-efficiency circuit than the complex closed-loop amplifier in the design of pipelined ADCs [4].

\section{CiRcuit Details}

\section{A. Track-and-Hold Amplifier}

The high-speed THA reduces the errors of clock skew and enhances the conversion rate preceding the first-stage comparators. Shown in Fig. 3, to reduce input-dependent turn-on resistance, the track-and-hold circuit adopts complementary MOS switches. Two dummy switches, $M_{3}$ and $M_{4}$, with half size of $M_{1}$ and $M_{2}$, absorb the input-dependent charge injection. CMOS switches and capacitors $\left(C_{H}\right)$ sample the input signal. Then, nMOS source followers act as unity-gain buffers. The sources of $M_{5}-M_{8}$ are tied to their own bulks by the deep-N-well process to eliminate body effect and reduce the threshold voltage. To obtain better matching and reduce clock feedthrough, $C_{H}$ is conservatively chosen as $300 \mathrm{fF}$. Two interleaved THAs controlled by nonoverlapped clocks, $\phi_{1}$ and $\phi_{2}$, sample the input signal for each bank of the pipelined stages.

\section{B. Pipelined Stage With Open-Loop Amplifiers}

The conventional flip-around radix-2 1.5-bit pipelined stage using a closed-loop amplifier is shown in Fig. 4(a). The multiplying DAC (MDAC) consists of a high-gain amplifier and capacitors, $C_{F}$ and $C_{S}$. When clock $\phi_{1}$ is high, $S_{1 a}, S_{1 b}, S_{1 c}$ are $O N$ and $S_{2 a}, S_{2 b}$ are $O F F, V_{i}$ is sampled to $C_{F}$ and $C_{S}$. The redundant-signed-digit (RSD) [9] code, $D_{i}$, which belongs to $[-1,0,+1]$, is evaluated by comparing $V_{i}$ with $V_{\mathrm{REF}} / 4$ and
$-V_{\mathrm{REF}} / 4$. When clock $\phi_{2}$ is high, $S_{1 a}, S_{1 b}, S_{1 c}$ are $O F F$ and $S_{2 a}, S_{2 b}$ are $O N$, the output $V_{i+1}$ is given by

$$
\begin{aligned}
V_{i+1}=\frac{1}{1+\frac{1}{A_{v}}} & \cdot \frac{C_{S}+C_{F}}{C_{F}} \\
& \times\left(\frac{C_{S}+C_{F}}{C_{F}} \times V_{i}-V_{\mathrm{REF}} \cdot \frac{C_{S}}{C_{F}} \times D_{i}\right)
\end{aligned}
$$

where $A_{v}$ is the open-loop gain of the amplifier. For the case with $A_{v}=\infty$ and $C_{F}=C_{S}$, (6) is reduced to

$$
V_{i+1}=2 \times V_{i}-V_{\mathrm{REF}} \times D_{i} .
$$

Shown in Fig. 4(b), the gain of the open-loop amplifier needs to be 2 to scale up the difference between $V_{i}$ and reference voltage. The operation of the pipelined stage with an ideal openloop amplifier can be expressed as

$$
V_{i+1}=2 \times\left(V_{i}-\frac{V_{\mathrm{REF}}}{2} \times D_{i}\right)
$$

which is identical to (7). When clock $\phi_{1}$ is high, $S_{1 a}, S_{1 b}$ are $O N$ and $S_{2}$ is $O F F$, input voltage $V_{i}$ is sampled at node $N$. When clock $\phi_{2}$ is high, $S_{1 a}, S_{1 b}$ are $O F F$ and $S_{2}$ is $O N$, node $N$ is now connected to $V_{\mathrm{REF}} / 2$ to perform the operation of (8). Fig. 4(c) shows the implementation of the differential open-loop pipelined stage, where only upper half switches and capacitor network are illustrated for simplicity. When clock $\phi_{1}$ is high, $S_{1 a}, S_{c 1}, S_{c 2}$, and $S_{c 3}$ are $O N$, and $S_{2 a}, S_{2 b}$, and $S_{2 c}$ are $O F F$, $V_{\text {in }}$ is sampled at $C_{S}$. When clock $\phi_{2}$ is high, $S_{1 a}, S_{c 1}, S_{c 2}$, and $S_{c 3}$ are $O F F$, and one of the three switches, $S_{2 a}, S_{2 b}$, and $S_{2 c}$, selected by the decoder is $O N$. Then, open-loop amplifier magnifies $V_{\text {in }}$ to $V_{\text {out }}$.

Designing an open-loop amplifier in pipelined ADCs is deliberated on three aspects.

1) Capacitive Attenuation: The parasitic capacitance of the input node of the open-loop amplifier attenuates the input voltage due to charge sharing. Shown in Fig. 5(a), when $V_{i}$ is sampled in $C_{S}$, and node $X$ is reset to ground, the parasitic capacitance, $C_{P}$, has no effect on the sampling mode. However, in amplification mode [Fig. 5(b)], the charge in $C_{S}$ is shared by $C_{P}$, and the voltage of node $X$ can be derived as

$$
V_{X}=-\left(\frac{C_{S}}{C_{S}+C_{P}}\right)\left(V_{i}-\frac{V_{\mathrm{REF}}}{2} \times D_{i}\right) \text {. }
$$

Therefore, the open-loop amplifier must compensate the attenuation of $C_{S} /\left(C_{S}+C_{P}\right)$.

2) Circuit Elaboration: Fig. 6(a) shows a differential amplifier with source degeneration. The small-signal model in (10) can be approximated to the amplifier's behavior:

$$
V_{\mathrm{out}}=\frac{g_{m} R_{D}}{1+g_{m} R_{S}} V_{\mathrm{in}}
$$

if the body effect is neglected. The source degeneration resistor $R_{S}$ improves the linearity of the amplifier. Although the nominal gain of the open-loop amplifier is 2 , the overall gain with capacitor networks is altered by the parasitic capacitive voltage division. To compensate the unknown gain reduction, the gain of the amplifier itself must be tunable. From (10), the voltage 


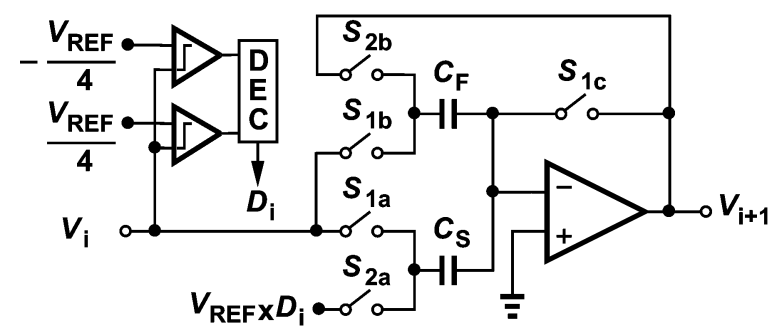

(a)

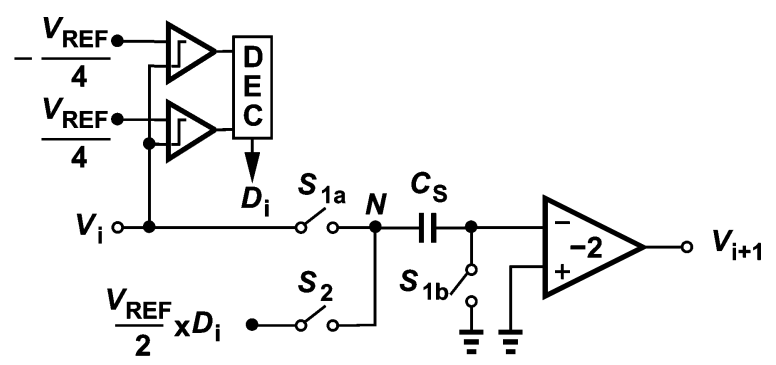

(b)

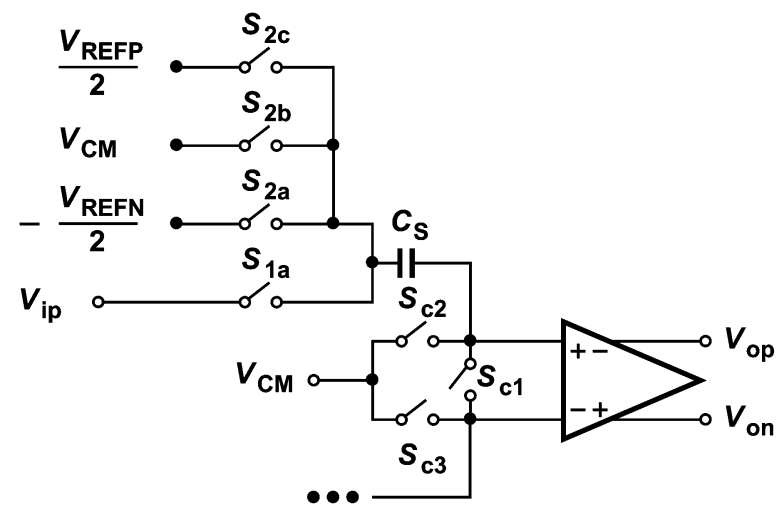

(c)

Fig. 4. (a) Conventional pipelined stage. (b) Pipelined stage with an open-loop amplifier. (c) Differential implementation of (b).

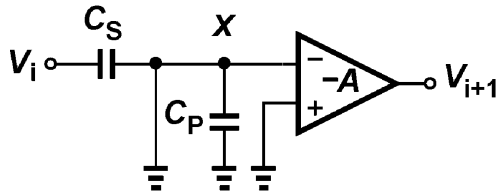

(a)

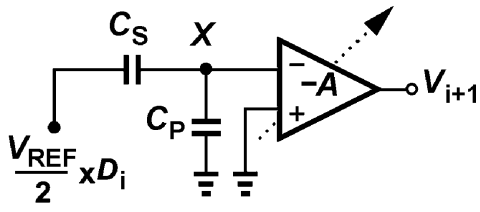

(b)

Fig. 5. Open-loop amplifier (a) in sampling mode and (b) in amplification mode.

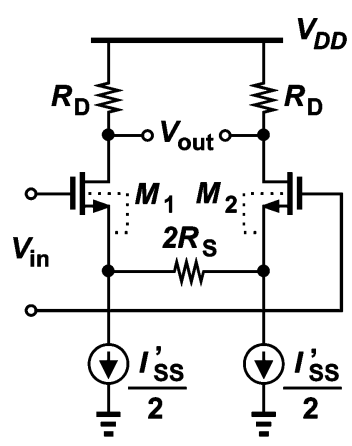

(a)

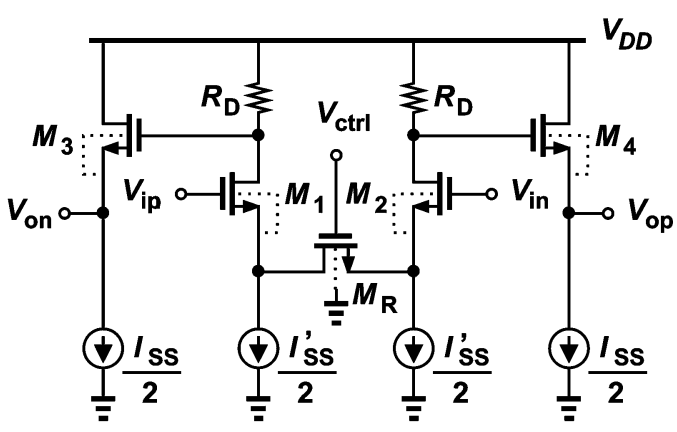

(b)

Fig. 6. (a) Differential amplifier with source degeneration. (b) Open-loop amplifier.

gain of the open-loop amplifier is a function of $g_{m}, R_{D}$ and $R_{S}$. Changing $g_{m}$ by tuning the bias current or $R_{D}$ will affect the DC bias point, and may even turn the amplifier $O F F$. Thus, $R_{S}$ becomes the best variable to adjust the gain of amplifier. Shown in Fig. 6(b), a triode-region nMOS transistor, $M_{R}$, replaces the degeneration resistor for gain tuning by adjusting the gate voltage, $V_{\text {ctrl }}$. Both bulks of $M_{1}$ and $M_{2}$ are connected to their sources to eliminate the body effect. The source followers, $M_{3}$ and $M_{4}$, not only shift DC level of the amplifier but also provide buffering to extend the operating speed of the gain stage. Furthermore, the source follower shifts the output voltage down by $\left(V_{\mathrm{TH}}+V_{\mathrm{ov}}\right)$, where $V_{\mathrm{TH}}$ is the threshold voltage and $V_{\mathrm{OV}}$ is the over-drive voltage. Then, the input common-mode voltage can be designed to yield the same output common-mode voltage.

3) Linearity Effect: The differential open-loop amplifier in the gain stage exhibits an "odd-symmetric" input/output charac- 


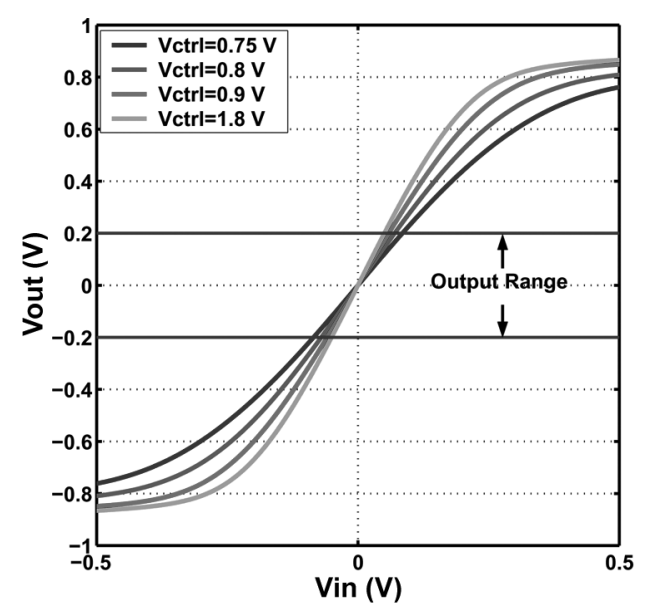

(a)

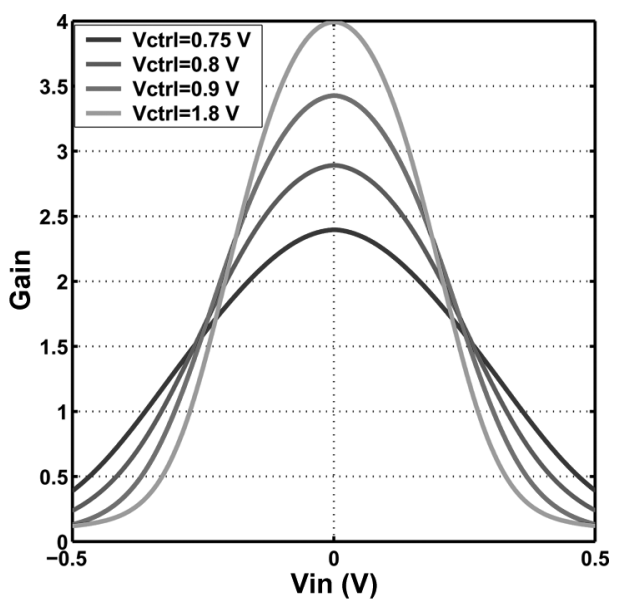

(b)

Fig. 8. Open-loop amplifier. (a) Input-output transfer curves. (b) Corresponding gains.

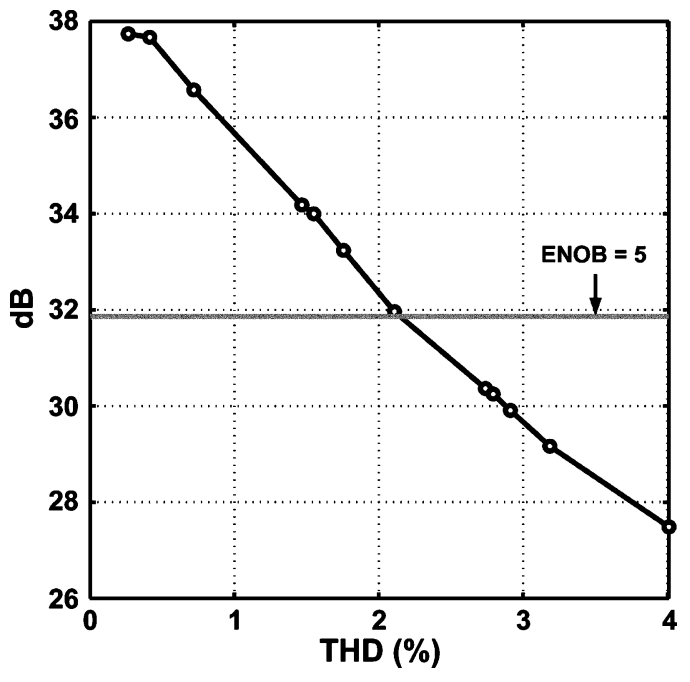

Fig. 7. Simulated SNDR of ADC versus THD of open-loop amplifier.

teristic. The Taylor expansion of the characteristic in the range of interest can be expressed as

$$
y(t)=\alpha_{1} x(t)+\alpha_{3} x^{3}(t)+\alpha_{5} x^{5}(t)+\cdots .
$$

Because the cubic term of (11) dominates the linearity of the ADC's performance, the higher order terms in the behavioral model are neglected for simplicity. Fig. 7 shows the signal-tonoise-plus-distortion ratio (SNDR) of the 6-bit ADC versus total harmonic distortion (THD) of the gain stage, where $\alpha_{1}$ is equal to two, $\alpha_{3}$ is varied from 0 to -33 , and the amplitude of input signal is 0.2 [10]. The simulation indicates that less than $2.1 \%$ THD is required in the open-loop amplifier for the proposed 6-bit ADC.

Fig. 8(a) and (b) shows HSpice simulation results of the open-loop amplifier input/output transfer curves and their corresponding gains for different $V_{\text {ctrl }}$. Parameters in this design are $R_{D}=500 \Omega, g_{m}=9 \mathrm{~mA} / \mathrm{V}$, and $R_{S}=11 \sim 150 \Omega$. By performing curve fitting for $V_{\text {out }}$ between $-0.2 \mathrm{~V}$ and $0.2 \mathrm{~V}$, their parameters are shown in Table I, where $G_{\text {att }}$ represents
TABLE I

CURVE-FITTING PARAMETERS AND SimUlation REsults

\begin{tabular}{ccccccc}
\hline$V_{c t r l}$ & $\alpha_{1}$ & $\alpha_{3}$ & $G_{a t t}$ & $\alpha_{3} G_{a t t}^{3}$ & SNDR & THD \\
\hline $0.75 \mathrm{~V}$ & 2.39 & -5.83 & 0.85 & -3.60 & $37.26 \mathrm{~dB}$ & $0.43 \%$ \\
$0.80 \mathrm{~V}$ & 2.89 & -8.26 & 0.70 & -2.86 & $37.35 \mathrm{~dB}$ & $0.42 \%$ \\
$0.90 \mathrm{~V}$ & 3.43 & -11.42 & 0.59 & -2.34 & $37.40 \mathrm{~dB}$ & $0.39 \%$ \\
$1.80 \mathrm{~V}$ & 3.99 & -16.07 & 0.51 & -2.08 & $37.44 \mathrm{~dB}$ & $0.37 \%$ \\
\hline
\end{tabular}

the attenuation factor, $V_{\text {in }}$ is the input voltage before capacitive attenuation, and $V_{\text {out }}$ is the amplifier output. From (11), the output of the amplifier is expressed as

$$
\begin{aligned}
V_{\text {out }} & =\alpha_{1} \cdot G_{\text {att }} V_{\text {in }}+\alpha_{3} \cdot\left(G_{\text {att }} V_{\text {in }}\right)^{3} \\
& =\alpha_{1} G_{\text {att }} \cdot V_{\text {in }}+\alpha_{3} G_{\text {att }}^{3} \cdot V_{\text {in }}^{3} .
\end{aligned}
$$

Table I indicates that higher $V_{\text {ctrl }}$ results in larger $\alpha_{3}$. However, the overall coefficient of cubic term is attenuated by $G_{\text {att }}^{3}$ such that the output swing is constant. If the gain of the open-loop amplifier needs to be high, it means the effective input swing is small. Therefore, the nonlinearity is softened by the attenuation. Behavioral simulation results of the proposed ADC in Table I show that the performance is quite constant for the tunable control voltage range.

\section{Comparator}

To reduce static power consumption, this ADC applies dynamic comparators in the pipelined stage. Shown in Fig. 9, the comparator consists of an input differential pair $M_{1}-M_{2}$ and a back-to-back inverter latch $M_{3}-M_{6}$. Input parasitic capacitances of the differential pair will result in the memory effect of previous input value, thereby affecting the detection of the comparison. To eliminate this effect, switches reset these nodes when comparators are in sampling mode. The outputs of the dynamic comparator pass through a static latch to eliminate glitch and reduce sensitivity to meta-stability. Accordingly, the compared results are decoded into the digital code and control signals of switches in pipelined stages. 


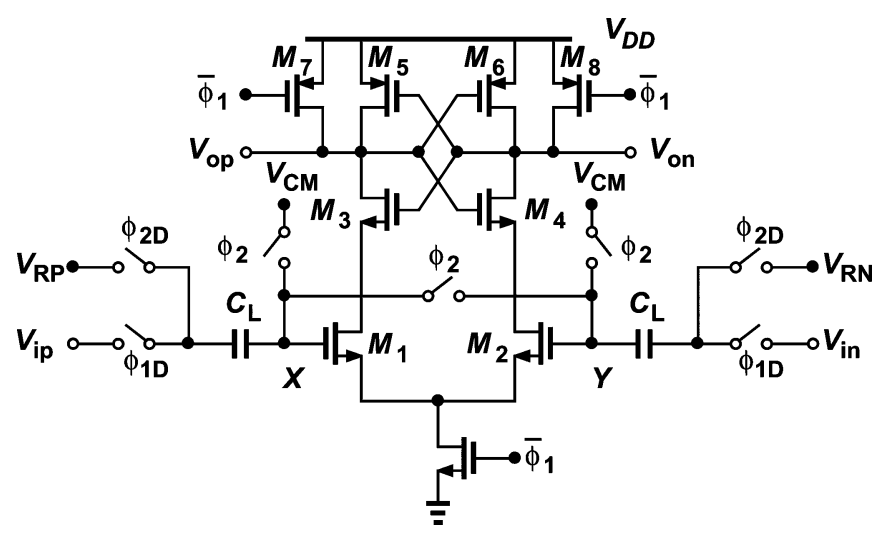

Fig. 9. Comparator.

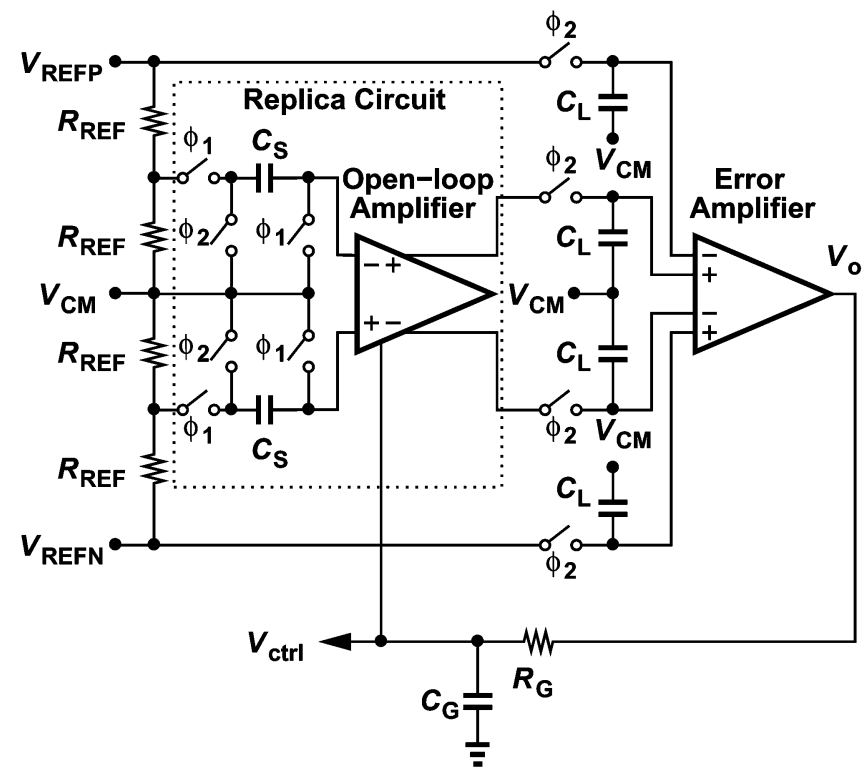

(a)

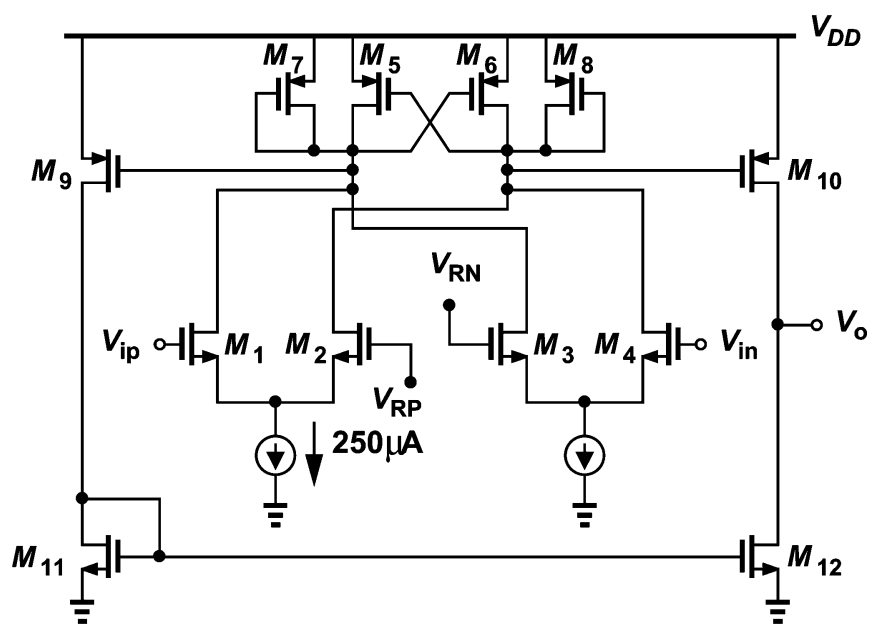

(b)

Fig. 10. (a) Gain-control servo loop. (b) Error amplifier.

\section{Global Gain Control}

Because the parasitic capacitance cannot be modeled precisely, a servo loop, shown in Fig. 10(a), is required to com-

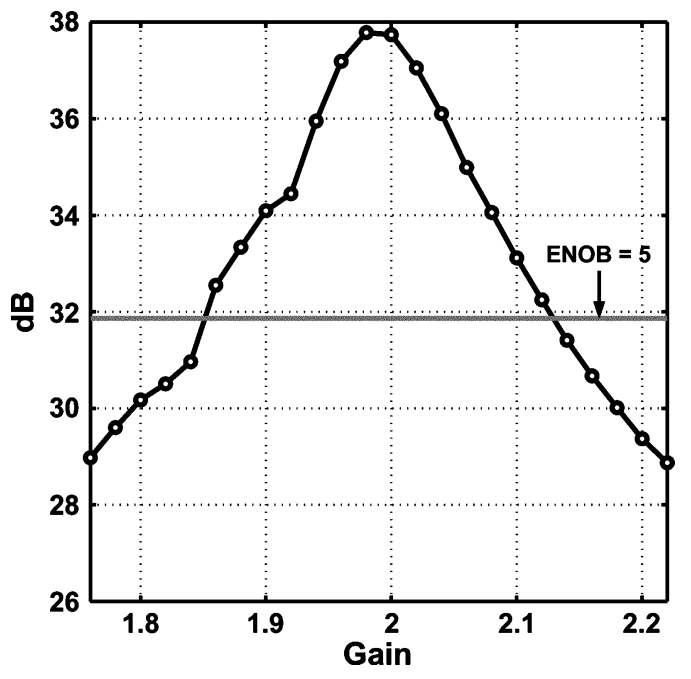

Fig. 11. Behavioral simulation of SNDR versus stage gain.

pensate the gain error. The duplicated residue amplifier serves as a replica circuit for the pipelined stages in this ADC. Since the layout of the residue amplifier circuit is identical to those in pipelined stages, these parasitic capacitances are approximately the same. The operation of the servo loop is as follows. The amplifier samples $V_{\mathrm{REF}} / 2$ at clock $\phi_{1}$. Then, the open-loop amplifier magnifies the sampled signal to obtain the output for comparison at clock $\phi_{2}$. Switches at the output of the open-loop amplifier are $O N$ during $\phi_{2}$ and are $O F F$ during $\phi_{1}$. Thus, these switches perform peak detection to sample the magnitude of the amplified value. Then, the sampled value is compared with $V_{\mathrm{REF}}$ by an error amplifier to obtain the amplified voltage error. With an external $R C$ low-pass loop filter, the control voltage $\left(V_{\text {ctrl }}\right)$ is fed back to the gate of $M_{R}$ in Fig. 6(b) for global gain control. Fig. 10(b) shows the schematic of the error amplifier. Since the external $R C$ low-pass filter (LPF) dominates the loop bandwidth, the error amplifier only acts as a gain stage to amplify the error signal. Thus, the settling time and phase margin of the error amplifier are not as stringent as in a normal operational amplifier. Consequently, the global gain control circuit only needs to operate in low frequency while open-loop amplifiers can operate at high-frequency conversion. Shown in Fig. 11, the behavioral simulation shows the gain error must be kept within 5\% to ensure 6-bit accuracy. The DC gain of the error amplifier is conservatively designed to about 46 $\mathrm{dB}$. An off-chip LPF with resistor $\left(R_{G}=30 \mathrm{k} \Omega\right)$ and capacitor $\left(C_{G}=20 \mathrm{pF}\right)$ suppresses the high-frequency components of the error amplifier output and presents the control signal to open-loop amplifiers. In HSpice simulation, the control signal settles within $380 \mathrm{~ns}$.

\section{E. Digital Error Correction and Decimation}

To provide a reliable digital output interface, the digital output is decimated to enable reliable acquisition by a logic analyzer. Since the pipelined ADC converts data at two interleaved banks, the decimation number must be odd to avoid sampling from the same bank. Based on these considerations, the decimation number is chosen as 21 . For example, if the original output sequence of Bankx and Banky is interleaved as 1, 2, 3,... 


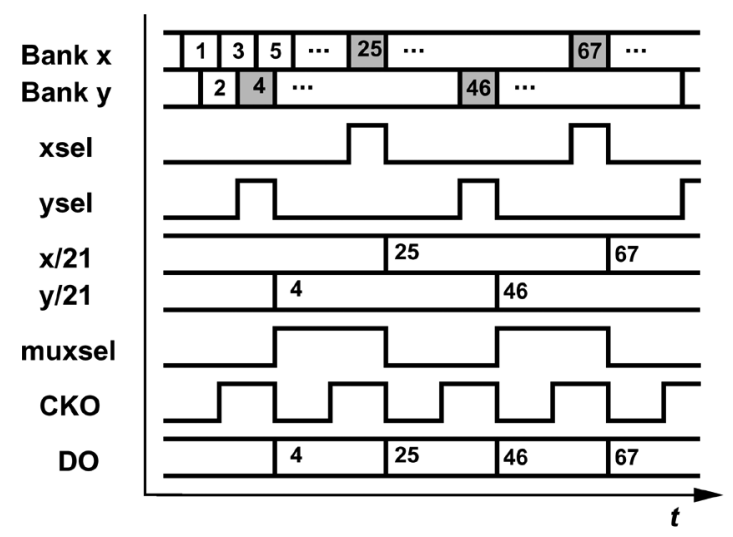

(a)

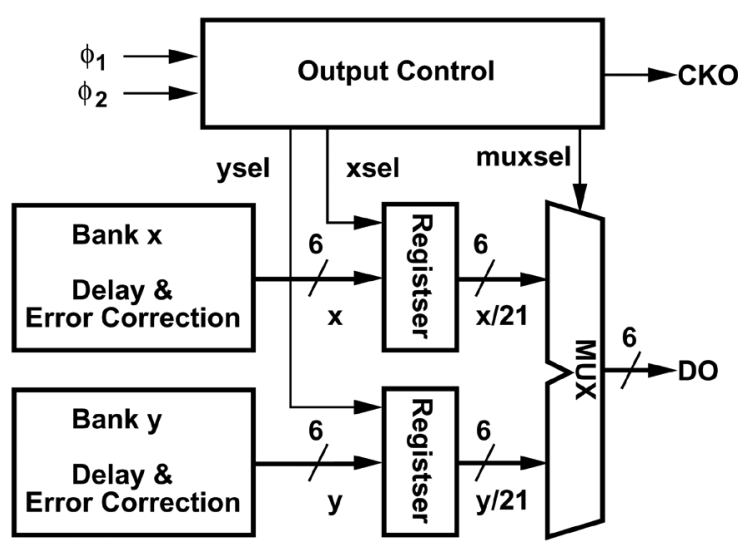

(b)

Fig. 12. (a) Decimation timing. (b) Decimation output block.

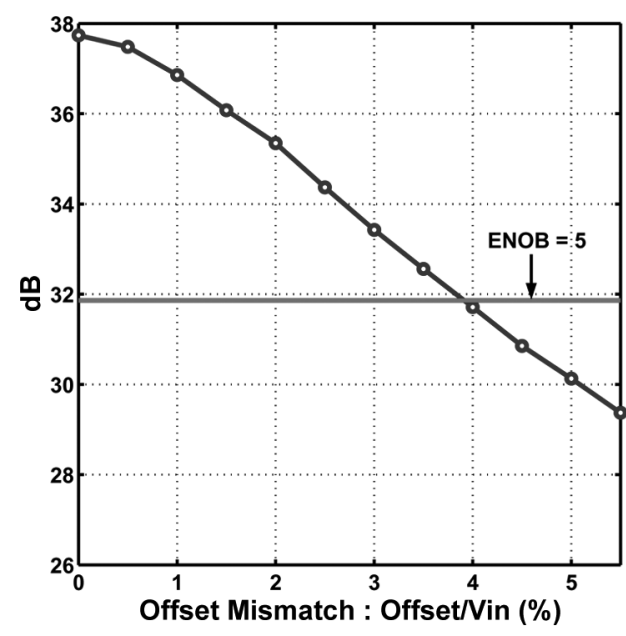

(a)

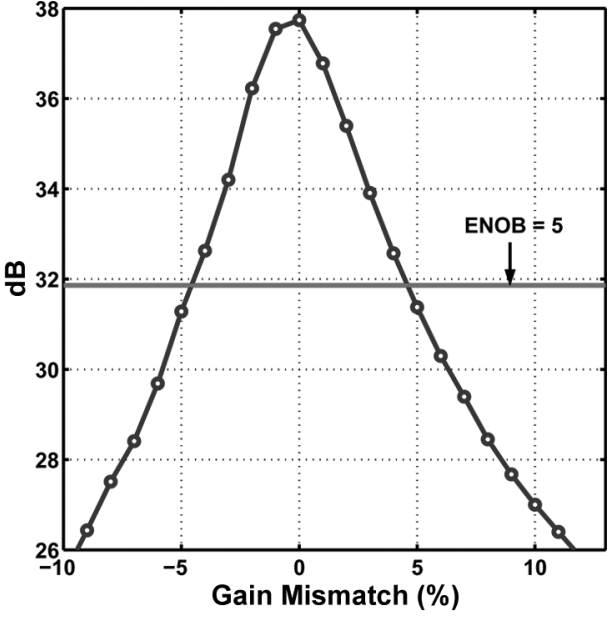

(b)

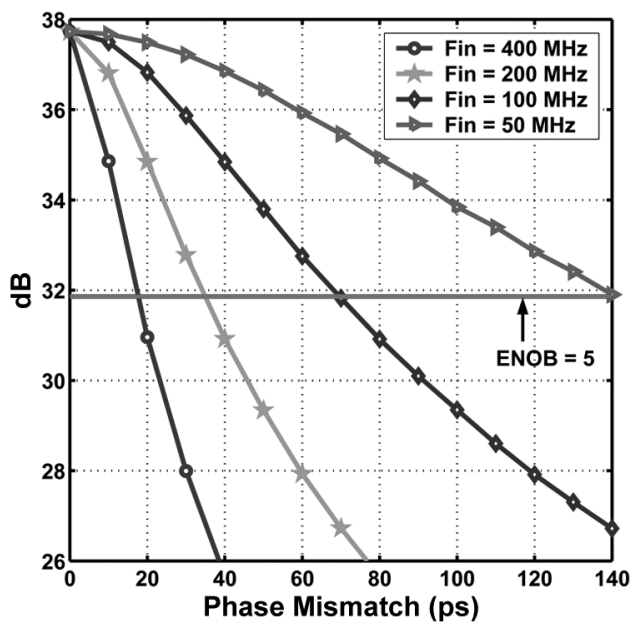

(c)

Fig. 13. Output SNDR for (a) offset mismatch, (b) gain mismatch, and (c) phase mismatch at 800 Msample/s.

in Fig. 12(a), the decimated sequence is $4,25,46,67, \ldots$ In order for the decimated output to keep the interleaved relationship, two signals, xsel and ysel, select the output of original sequence as $x / 21$ and $y / 21$ in Fig. 12(a). Finally, a control signal, muxsel, selects the inputs of the multiplexer to derive the output data, $D O$. The decimated clock, $C K O$, provides added convenience for data acquisition. Fig. 12(b) shows block diagram of corresponding output. All digital circuits are synchronous to avoid the need for accounting latency differences. 


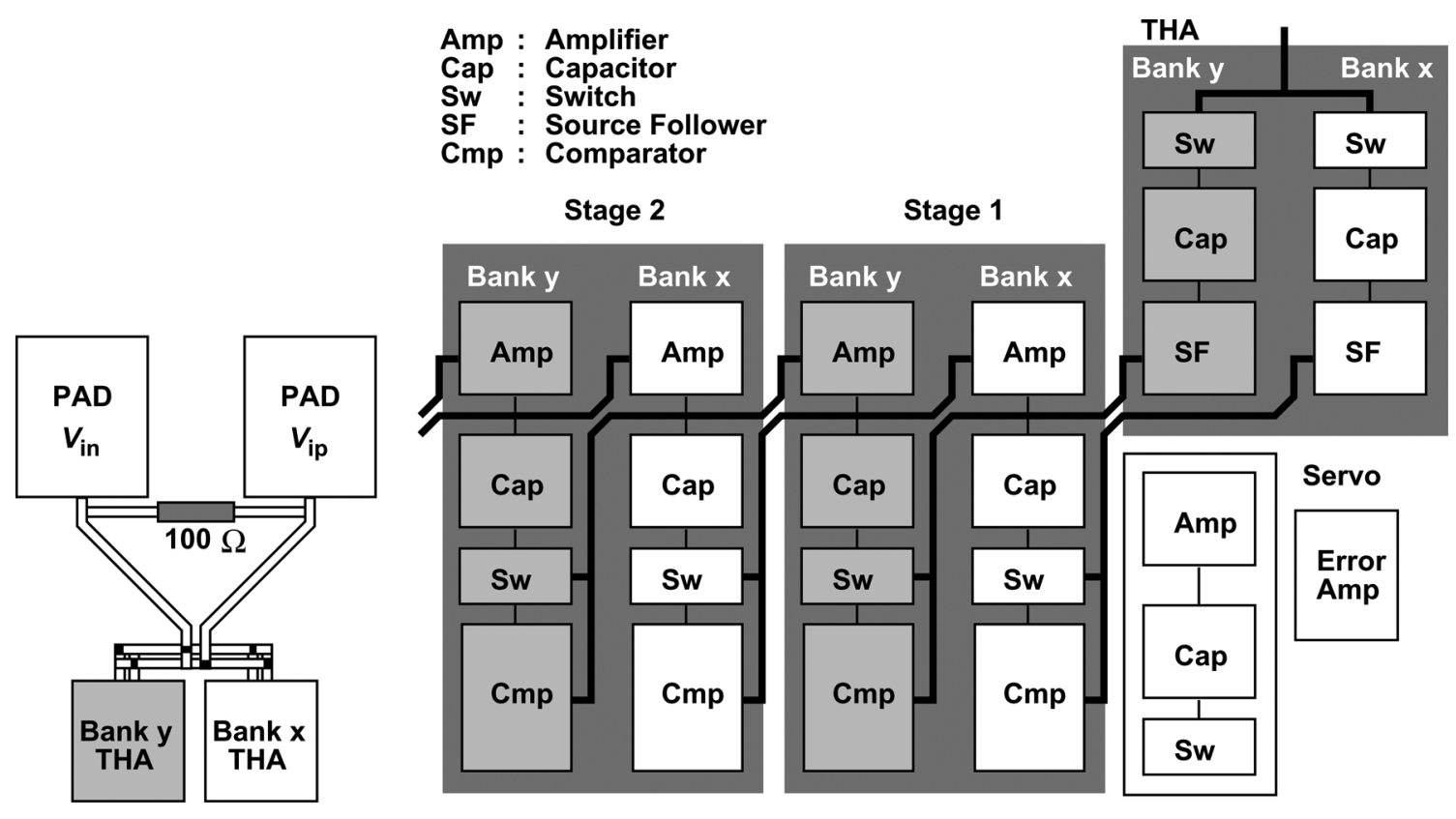

(a)

(b)

Fig. 14. (a) Layout between PADs and THAs. (b) Interleaved layout.

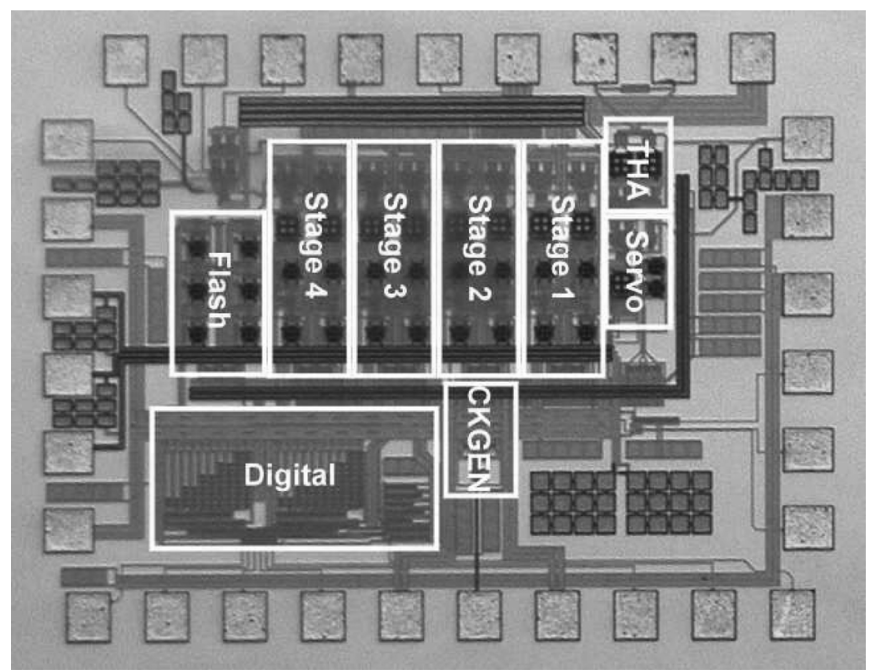

Fig. 15. ADC microphotograph.

\section{DESIGN CONSIDERATIONS}

The time-interleaved ADC is sensitive to mismatches in offset, gain, and phase [11]-[14]. In a 6-bit 800 Msamples/s pipelined ADC's behavioral simulation, the effects of mismatch in offset, gain, and phase are shown in Fig. 13. To achieve better than 5-bit performance, the offset, gain, and phase mismatch must be less than $3.8 \%$ of the full-scale input, $4.5 \%$, and $18 \mathrm{ps,}$ respectively.

In physical layout, the phase mismatch arises from different propagation delay between pads and input of two interleaved sampling capacitors. Shown in Fig. 14(a), the $100-\Omega$ resistor matches the output impedance of signal source to avoid the reflection. The routing between pads and the inputs of the two THAs is as short and symmetric as possible. Therefore, input
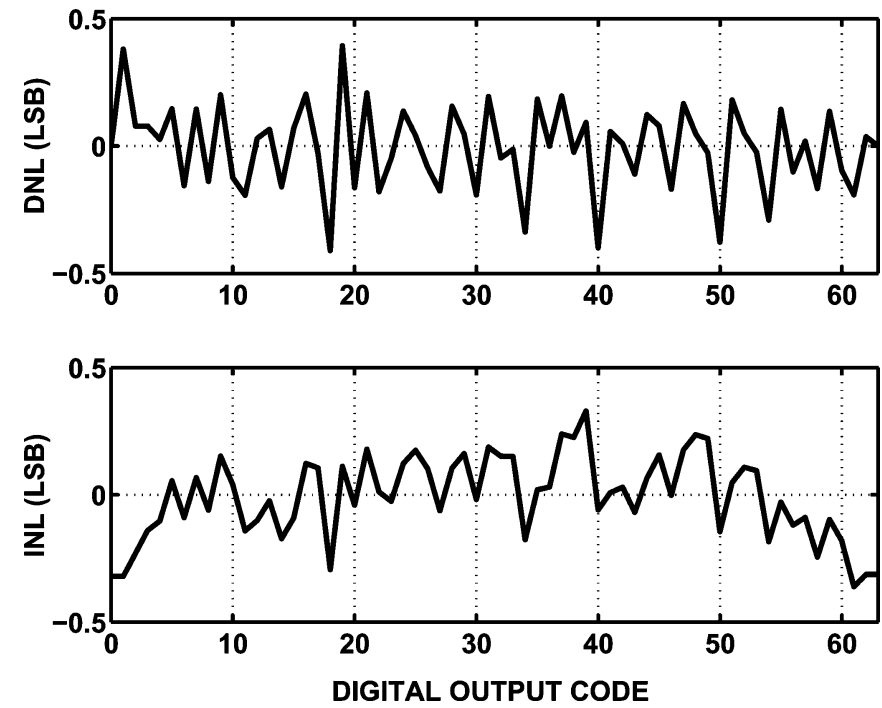

Fig. 16. Measured INL and DNL.

resistance including switches is approximately $100 \Omega$. With $300-\mathrm{fF}$ input capacitance, the time constant is about $30 \mathrm{ps}$ and the mismatch can be easily kept within 18 ps corresponding to the requirement in Fig. 13(c). In addition to input delay mismatch, the mismatch of two nonoverlapped clocks induces phase mismatches. Since the even duty cycle is important for interleaving, the input clock is divided by two to obtain the $50 \%$ duty cycle clock. In the layout, loading of two paths are balanced to meet the requirement. To alleviate the gain mismatch and offset mismatch, the two banks of pipelined stages interlace each other as shown in Fig. 14(b). The capacitors and guard rings isolate the analog part from noisy switching in this topology. 


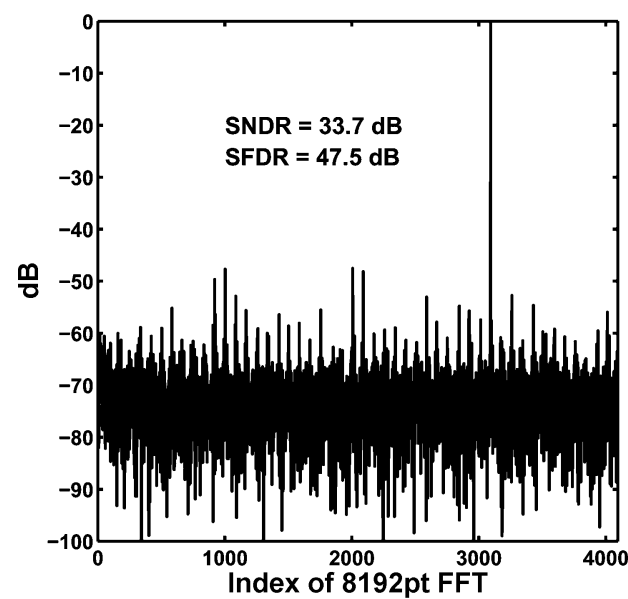

(a)

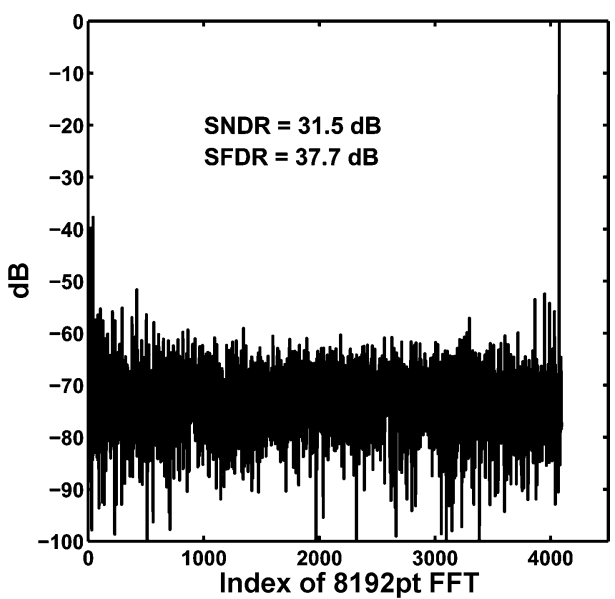

(b)

Fig. 17. (a) FFT of $100 \mathrm{MHz}$ at $800 \mathrm{Msample/s.} \mathrm{(b)} \mathrm{FFT} \mathrm{of} 399.9 \mathrm{MHz}$ at $800 \mathrm{Msample/s.}$

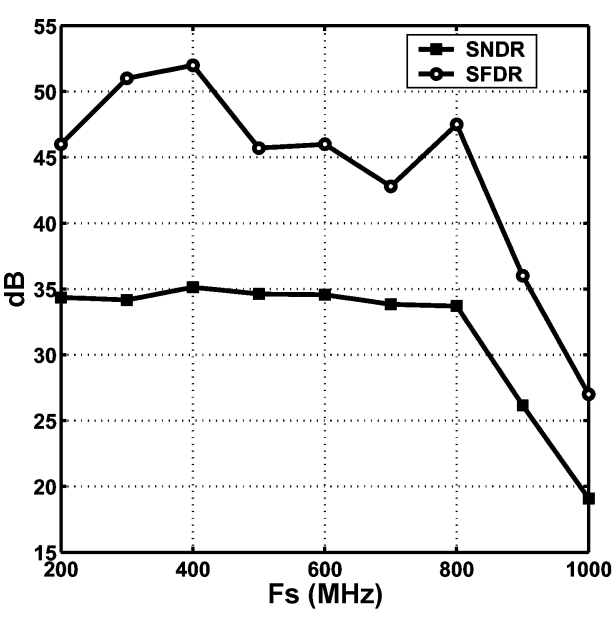

(a)

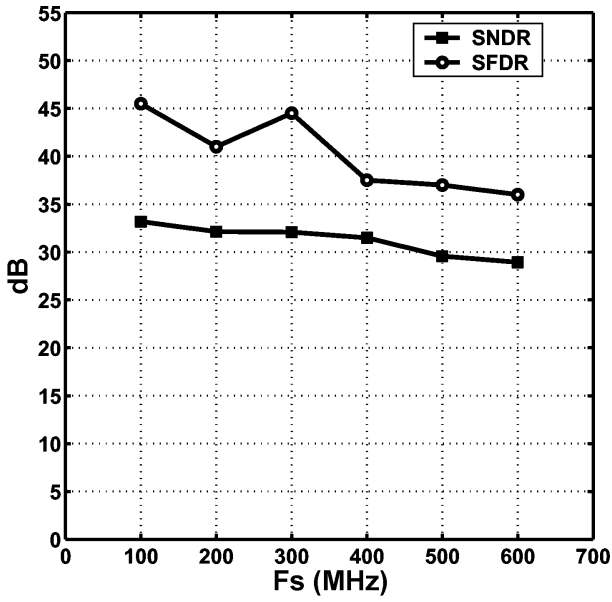

(b)

Fig. 18. (a) SNDR and SFDR versus conversion rate with $100 \mathrm{MHz}$ input. (b) SNDR and SFDR versus input frequency at $800 \mathrm{MS} / \mathrm{s}$.

IR drops of power lines in the layout affect gain accuracy of open-loop amplifiers. However, one advantage of pipelined ADCs is that gain-accuracy requirements in rear stages are lower than front stages. Thus, the replica circuit in the servo loop is placed near the first stage to lower the sensitivity of gain variation. The power lines are composed of metal 4 and metal 6 with the width of $20 \mu \mathrm{m}$ in parallel, and the power pads are near the first stage. Because metal 6 is a thick layer, power-line resistance across the pipelined ADC is within $0.3 \Omega$. In addition, current routing of local bias reduces the problems with mismatch and power-line resistance by reducing the distance in the current mirror [15].

\section{EXPERIMENTAL RESULTS}

Fig. 15 shows the microphotograph of the ADC chip. This chip occupies $1 \times 1.2 \mathrm{~mm}^{2}$ and the active area is $0.5 \mathrm{~mm}^{2}$. It has been fabricated in a $0.18-\mu \mathrm{m}$ CMOS technology. Power lines of analog, digital, clock, and output blocks are separated and connected to on-chip decoupling capacitors to avoid the wire bouncing and noise coupling. Only one $1.8-\mathrm{V}$ power supply is required for this chip.
In high sample-rate systems a clean, low-jitter clock source must be employed. The input signal amplitude is $400 \mathrm{mVpp}$, input common-mode voltage is $1.3 \mathrm{~V}$, and the decimated output is $D O$, as depicted in Fig. 12(a).

Integral nonlinearity (INL) and differential nonlinearity (DNL) are calculated based on code-density measurement on the combined signal from both channels in Fig. 16. With a $100-\mathrm{MHz}$ sinusoidal signal at $800-\mathrm{Msample} / \mathrm{s}$ conversion rate, peak INL is $0.36 \mathrm{LSB}$, and peak DNL is $0.41 \mathrm{LSB}$. Fig. 17 shows the output spectrum of the converted data at $800 \mathrm{Msample} / \mathrm{s}$. The spectrum of the sampled signal is folded between 0 to $19 \mathrm{MHz}$ because of decimation by 21 . With a $100-\mathrm{MHz}$ input, SNDR and spurious-free dynamic range (SFDR) of the ADC are $33.7 \mathrm{~dB}$ and $47.5 \mathrm{~dB}$, respectively. Near Nyquist sampling, SNDR and SFDR of the ADC still maintain above $31.5 \mathrm{~dB}$ and $37.5 \mathrm{~dB}$, respectively.

The ADC's dynamic performance is measured by varying the sampling rate from $200 \mathrm{Msample/s}$ to 1 Gsample/s. Shown in Fig. 18(a), with the input signal frequency of $100 \mathrm{MHz}$, the effective number of bits (ENOB) is better than 5.3 up to $800 \mathrm{Msample} / \mathrm{s}$. In higher conversion rate, the output voltage of the open-loop amplifier cannot settle within the desired level. 
TABLE II

6-bit ADC FOM COMPARASION

\begin{tabular}{|c|c|c|c|c|c|c|c|}
\hline Author/year & Architecture & $\begin{array}{c}\text { Technology } \\
(\mu \mathrm{m})\end{array}$ & $\begin{array}{c}\text { Fs } \\
(\mathrm{MHz})\end{array}$ & $\begin{array}{c}\text { ENOB } \\
(\mathrm{bit})\end{array}$ & $\begin{array}{c}\text { ERBW } \\
(\mathrm{MHz})\end{array}$ & $\begin{array}{c}\text { Power } \\
(\mathrm{mW})\end{array}$ & $\begin{array}{c}\text { FOM } \\
(\mathrm{pJ} / \mathrm{step})\end{array}$ \\
\hline Sander'05 [17] & Flash & 0.13 & 1200 & 5.7 & 700 & 160 & 2.2 \\
Jiang'05 [3] & Interleaved Flash & 0.18 & 2000 & 5.55 & 941 & 310 & 3.5 \\
Draxelmayr'04 [7] & Interleaved SAR & 0.09 & 600 & 5.35 & 300 & 10 & 0.4 \\
Uyttenhove'03 [18] & Flash & 0.25 & 1300 & 5.35 & 550 & 600 & 13.4 \\
Scholtens'02 [19] & Flash & 0.18 & 1600 & 5.7 & 610 & 328 & 5.17 \\
Geelen'01 [16] & Flash & 0.35 & 900 & 5.65 & 450 & 200 & 4.4 \\
Choi'01 [2] & Flash & 0.35 & 1000 & 5.7 & 650 & 500 & 7.4 \\
This work & Interleaved Pipelined & 0.18 & 800 & 5.35 & 460 & 105 & 2.8 \\
\hline
\end{tabular}

TABLE III

PERFORMANCE SUMMARY

\begin{tabular}{|l|l|}
\hline Technology & TSMC 0.18 $\mu \mathrm{m}$ CMOS \\
\hline Chip Area / Active Area & $1.2 \mathrm{~mm}^{2} / 0.5 \mathrm{~mm}^{2}$ \\
\hline Resolution & 6 bits \\
\hline Conversion Rate & $800 \mathrm{MS} / \mathrm{s}$ \\
\hline INL / DNL & $0.36 \mathrm{LSB} / 0.41 \mathrm{LSB}$ \\
\hline Input Range & $400 \mathrm{mV}$-p differential \\
\hline SFDR @ fin=100 MHz & $47.5 \mathrm{~dB}$ \\
\hline SNDR @ fin=100 MHz & $\begin{array}{l}33.7 \mathrm{~dB} \\
\mathbf{3 1 . 5} \mathrm{dB}\end{array}$ \\
\hline Power Dissipation & $\begin{array}{l}\text { analog }: 75 \mathrm{~mW} \\
\text { digital }: 30 \mathrm{~mW}\end{array}$ \\
\hline Supply Voltage & $1.8 \mathrm{~V}$ \\
\hline
\end{tabular}

Therefore, the global gain control mechanism must increase the voltage gain of the amplifier. When the required gain is beyond the control range of servo loop, the performance degrades. Accordingly, the SNDR of the ADC descends at the sampling frequency over $800 \mathrm{Msample/s}$. When the sampling rate is fixed at $800 \mathrm{Msample/s,} \mathrm{SNDR} \mathrm{and} \mathrm{SFDR} \mathrm{with} \mathrm{various}$ input frequencies are plotted in Fig. 18(b). Since SNDR and SFDR drop slightly above Nyquist frequency, the performance is quite constant for the input signals with wide frequency range. Excluding output buffers, this ADC consumes $75 \mathrm{~mW}$ for analog circuits and $30 \mathrm{~mW}$ for the rest of digital circuits at $800 \mathrm{Msample} / \mathrm{s}$. The figure of merit (FOM) calculated by the definition of (13) [16] is $2.8 \mathrm{pJ}$.

$$
\mathrm{FOM}=\frac{\text { Power }}{2^{\mathrm{ENOB}} \cdot 2 \cdot \mathrm{ERBW}}
$$

where ERBW is effective resolution bandwidth. Table II shows the FOM comparison of 6-bit ADCs. The measured performance summary is shown in Table III.

\section{CONCLUSION}

The combination of voltage-mode open-loop amplifiers and two-bank interleaving in pipelined architecture realizes a 6-bit pipelined ADC achieving $800 \mathrm{Msample/s}$ without the need of digital calibration. The servo loop of global gain control compensates the gain attenuation by parasitic capacitances. Applying global gain control relaxes the stringent requirement of high-gain amplifier design and allows the pipelined ADC with open-loop amplifiers to operate at high-conversion rate. This approach extends the conversion speed of designing a pipelined ADC in low-voltage and low-power applications for nano-meter technologies.

\section{ACKNOWLEDGMENT}

The authors would like to thank Chip Implementation Center (CIC) for chip fabrication.

\section{REFERENCES}

[1] A. Varzaghani and C.-K. K. Yang, "A $600 \mathrm{Ms} / \mathrm{s}$ 5-bit pipelined analog-to-digital converter for serial-link applications," in Symp. VLSI Circuits Dig. Tech. Papers, 2004, pp. 276-279.

[2] M. Choi and A. A. Abidi, "A 6-bit 1.3-Gsample/s A/D converter in 0.35- $\mu \mathrm{m}$ CMOS," IEEE J. Solid-State Circuits, vol. 36, no. 12, pp. 1847-1858, Dec. 2001.

[3] X. Jiang and M.-C. Frank, "A 1-GHz signal bandwidth 6-bit CMOS with power efficient averaging," IEEE J. Solid-State Circuits, vol. 40, no. 2, pp. 532-535, Feb. 2005.

[4] B. Murmann and B. E. Boser, "A 12-bit $75 \mathrm{Ms} / \mathrm{s}$ pipelined ADC using open-loop residue amplifier," IEEE J. Solid-State Circuits, vol. 38, no. 12, pp. 2040-2050, Dec. 2003.

[5] K. Poulton, R. Neff, A. Muto, W. Liu, A. Burstein, and M. Heshami, "A 4 Gsample/s 8b ADC in $0.35 \mu \mathrm{m}$ CMOS," in IEEE ISSCC Dig. Tech. Papers, 2002, vol. , pp. 166-457.

[6] K. Poulton, R. Neff, B. Setterberg, B. Wuppermann, T. Kopley, R. Jewett, J. Pernillo, C. Tan, and A. Montijo, "A 20 Gs/s 8 b ADC with a $1 \mathrm{MB}$ memory in $0.18 \mu \mathrm{m}$ CMOS," in IEEE ISSCC Dig. Tech. Papers, 2003, vol. , pp. 318-319.

[7] D. Draxelmay, "A 6 b $600 \mathrm{MHz} 10 \mathrm{~mW}$ ADC array in digital $90 \mathrm{~nm}$ CMOS," in IEEE ISSCC Dig. Tech. Papers, 2004, pp. 264-265.

[8] J. Goes, J. C. Vital, and J. E. Franca, "Systematic design for optimization of high-speed self-calibrated pipelined A/D converters," IEEE Trans. Circuits Syst. II: Analog Digit. Signal Process., vol. 45, no. 12, pp. 1513-1526, Dec. 1998. 
[9] B. Ginetti, P. G. A. Jespers, and A. Vandemeulebroecke, "A CMOS 13-bit cyclic RSD A/D converter," IEEE J. Solid-State Circuits, vol. 27, no. 7, pp. 957-965, Jul. 1992.

[10] B. Razavi, Design of Analog CMOS Integrated Circuits. New York: McGraw-Hill, 2001.

[11] C. S. G. Conroy, D. W. Cline, and P. R. Gray, "An 8-bit 85-ms/s parallel pipeline A/D converter in 1- $\mu$ m CMOS," IEEE J. Solid-State Circuits, vol. 28, no. 4, pp. 447-454, Apr. 1993.

[12] D. Fu, K. C. Dyer, S. H. Lewis, and P. J. Hurst, "A digital background calibration technique for time-interleaved analog-to-digital converters," IEEE J. Solid-State Circuits, vol. 33, no. 12, pp. 1904-1911, Dec. 1998.

[13] K. C. Dyer, D. Fu, S. H. Lewis, and P. J. Hurst, "An analog background calibration technique for time-interleaved analog-to-digital converters," IEEE J. Solid-State Circuits, vol. 33, no. 12, pp. 1912-1919, Dec. 1998

[14] B. Xia, A. Valdes-Garcia, and E. Sanchez-Sinencio, "A 10-bit 44-Ms/s $20 \mathrm{~mW}$ configurable time-interleaved pipeline ADC for a dual-mode 802.11b/Bluetooth receiver," IEEE J. Solid-State Circuits, vol. 41, no. 3, pp. 530-539, Mar. 2006

[15] P. R. Gray, P. J. Hurst, S. H. Lewis, and R. G. Meyer, Analysis and Design of Analog Integrated Circuits, 4th ed. New York: Wiley, 2001.

[16] G. Geelen, "A 6 b 1.1 Gsample/s CMOS A/D converter," in IEEE ISSCC Dig. Tech. Papers, 2001, pp. 128-129.

[17] C. Sandner, M. Clara, A. Santner, T. Hartig, and F. Kuttner, "A 6-bit $1.2-\mathrm{Gs} / \mathrm{s}$ low-power flash-ADC in $0.13-\mu \mathrm{m}$ digital CMOS," IEEE $J$. Solid-State Circuits, vol. 40, no. 7, pp. 1499-1505, Jul. 2005.

[18] K. Uyttenhove and M. S. J. Steyaert, "A 1.8-V 6-bit 1.3-GHz flash ADC in 0.25- $\mu \mathrm{m}$ CMOS," IEEE J. Solid-State Circuits, vol. 38, no. 7, pp. 1115-1122, Jul. 2003.

[19] P. C. S. Scholtens and M. Vertregt, "A 6-bit 1.6-Gsample/s flash ADC in $0.18-\mu \mathrm{m}$ CMOS using averaging termination," IEEE J. Solid-State Circuits, vol. 37, no. 12, pp. 1599-1609, Dec. 2002.

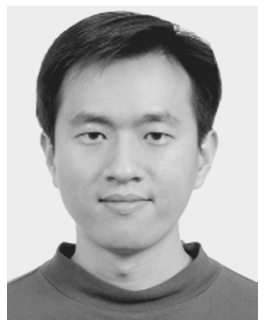

Ding-Lan Shen (S'06) was born in Taiwan, R.O.C., in 1970. He received the B.S. and M.S. degrees from National Sun Yat-Sen University, Taiwan, in 1994 and 1996, respectively. From 1998 to 2002, he was an Optical-Servo Engineer with ALi Corporation. $\mathrm{He}$ is currently working toward the Ph.D. degree at the Graduate Institute of Electronics Engineering (GIEE), National Taiwan University.

His research interests include high-speed $\mathrm{A} / \mathrm{D}$ and D/A converters, converter calibration algorithms, and mixed-signal circuits design.

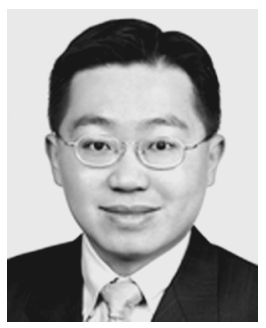

Tai-Cheng Lee (S'91-M'95)was born in Taiwan, R.O.C., in 1970. He received the B.S. degree from National Taiwan University in 1992, the M.S. degree from Stanford University in 1994, and the Ph.D. degree from the University of California, Los Angeles, in 2001, all in electrical engineering.

He worked for LSI logic from 1994 to 1997 as a Circuit Design Engineer. He served as an Adjunct Assistant Professor at the Graduate Institute of Electronics Engineering (GIEE), National Taiwan University, from 2001 to 2002. Since 2002, he has been with the Electrical Engineering Department and GIEE, National Taiwan University, where he is an Associate Professor. His research interests include data converters, PLL systems, and RF circuits. 